Article type : Mini commentary

Mini-commentary on BJOG-19-0881.R1: Endometriosis Fertility Index for Predicting Non-assisted Reproductive Technology Pregnancy after Endometriosis Surgery: A Systematic Review and Meta-analysis

\title{
Why and when you should use the Endometriosis Fertility Index (EFI)
}

\section{Carla Tomassetti}

University Hospitals Leuven, Dept. Obstetrics and Gynaecology, Leuven University Fertility Center

KU Leuven, Faculty of Medicine, Dept. Development and Regeneration, Division Woman and Child

carla.tomassetti@uzleuven.be

Since the eighties, the revised ASRM classification (American Society of Reproductive Medicine, the former American Fertility Society) has been used to surgically stage the severity of endometriosis. However, the correlation between the rASRM stage and the likelihood of pregnancy following therapy (including surgery) is very poor, rendering its usefulness as a clinical instrument for patient management and counselling in endometriosis-associated infertility futile. In 2010, Adamson and Pasta (Adamson and Pasta. Fertil Steril 2010;94:1609-1615)) developed the Endometriosis Fertility Index (EFI), a score ranging from 0 to 10 points. The EFI was presented as an end-of-surgery scoring system that predicts pregnancy rates without the use of ART (assisted reproductive technology) treatment in patients operated for endometriosis who also suffer from infertility, and takes into account all endometriosis rASRM stages. 5 out of 10 possible points are based on patient characteristics (age, duration of infertility and history of pregnancy), and the other 5 on surgical information: rASRM lesion score, rASRM total score, and a qualitative visual assessment by the surgeon of the adnexal function (the 'least function' score). As for any given staging system, apart from internal validation - as in the initial study by Adamson and

This article has been accepted for publication and undergone full peer review but has not been through the copyediting, typesetting, pagination and proofreading process, which may lead to differences between this version and the Version of Record. Please cite this article as doi: $\underline{10.1111 / 1471-0528.16180}$

This article is protected by copyright. All rights reserved 
Pasta - an extensive external validation is crucial for the EFI to become a clinically relevant instrument.

Vesali and colleagues now present in this issue (BJOG 2020 xxxx) a systematic review and metaanalysis - including 17 studies on 4598 women - to assess the accuracy of the EFI for predicting non-ART pregnancy. They provide a thorough overview of all external validation studies on the EFI and its correlation with non-ART (clinical) pregnancy, and are able to confirm the good performance of the EFI score in predicting the non-ART pregnancy rate, while acknowledging a substantial heterogeneity between studies.

Their findings, together with the fact that the EFI is also reliably reproducible (Tomassetti et al. BJOG 2020;127:107-114), further establish the EFI as one of the most important innovations in the past decade to optimize management of women with endometriosis-associated infertility, as the best (and only) objective scoring system available. In daily clinical practice, the EFI should therefore be used for postoperative management and counselling of patients on their reproductive options, i.e. for identifying those who may need subsequent ART or not. Indeed, using the EFI instead of the rASRM staging - regardless of endometriosis lesion type - provides a basis for deferring treatment with ART in the better prognostic groups on the one hand, and avoids wasting time with expectant management in patients with low EFI scores on the other hand. However, the place of the EFI in the decision-making process prior to surgery remains to be established.

From a research point of view, the good performance of the EFI implies that future studies on reproductive outcomes in endometriosis should report the EFI score (next to rASRM staging) as a clinical variable, not only to improve the design of clinical trials by better selecting and defining patient populations of interest, but also to enhance comparability of study findings and reduce heterogeneity when pooling data-analyses.

Disclosure of interests: Dr. Tomassetti reports grants from Research Foundation - Flanders (FWO), grants and other from Ferring Pharmaceuticals, grants and other from Merck SA, other from Lumenis, other from Nordic Pharma, other from Gedeon Richter, other from MSD, outside the submitted work. A completed disclosure of interest form is available to view online as supporting information.

This article is protected by copyright. All rights reserved 